\title{
DEGREES OF IRREDUCIBLE CHARACTERS AND NORMAL $p$-COMPLEMENTS
}

\author{
YA. G. BERKOVICH
}

(Communicated by Warren J. Wong)

\begin{abstract}
John Tate [1] proved that if $P \in \operatorname{Syl}_{p}(G), H$ is a normal subgroup of a finite group $G$ and $P \cap H \leq \Phi(P)(\Phi(G)$ is the Frattini subgroup of $G)$ then $H$ has a normal $p$-complement. We prove in this note that Tate's theorem has nice character-theoretic applications.
\end{abstract}

Theorem. Let $B$ be the intersection of the kernels of all nonlinear irreducible characters of $G$ with $p^{\prime}$-degree. Then $B \cap G^{\prime} \cap P \subseteq P^{\prime}$ where $P \in \operatorname{Syl}_{p}(G)$. Also, $B$ has a normal p-complement.

Proof. We suppose that $P_{0}=B \cap G^{\prime} \cap P \nless P^{\prime}$. Let $\operatorname{Lin}(P)$ be the set of all linear characters of $P$, and let $\lambda \in \operatorname{Lin}(P)$ satisfy $P_{0} \nless \operatorname{ker} \lambda$. Then the induced character $\lambda^{G}$ has degree $|G: P| \not \equiv 0(\bmod p)$. Let $\chi$ be an irreducible component of $\lambda^{G}$. Then $P_{0} \nless \operatorname{ker} \chi$ by Frobenius reciprocity. So $p$ divides $\chi(1)$ for all nonlinear irreducible components $\chi$ of $\lambda^{G}$. Since $p$ does not divide $\lambda^{G}(1)$, the character $\lambda^{G}$ has a linear component $\lambda^{0}$. Then $\lambda_{P}^{0}=\lambda$. Thus

$$
P \cap \operatorname{ker} \lambda^{0}=\operatorname{ker} \lambda \Varangle P_{0} \Rightarrow P_{0} \Varangle \operatorname{ker} \lambda^{0} .
$$

Since $G^{\prime} \leq \operatorname{ker} \lambda^{0}$, we have

$$
B \cap G^{\prime} \cap P=P_{0} \npreceq G^{\prime},
$$

which is a contradiction.

The last assertion follows from

Lemma. Let $P \in \operatorname{Syl}_{p}(G)$ and let $H \unlhd G$. If $H \cap G^{\prime} \cap P \leq P^{\prime}$, then $H$ has a normal p-complement.

Proof. Let $O^{p}(G)$ be the intersection of all $N \unlhd G$ such that $G / N$ is a $p$-group. Then $O^{p}(G)$ is characteristic in $G$. So $O^{p}(H) \unlhd G$ and

$$
O^{p}(H) \cap G^{\prime} \cap P \leq H \cap G^{\prime} \cap P \leq P^{\prime} .
$$

Received by the editors April 14, 1988 and, in revised form, July 19, 1988.

1980 Mathematics Subject Classification (1985 Revision). Primary 20C15, 20 D20.

In memory of Professor Samuil Davidovich Berman (Jan. 3, 1922-Feb. 18, 1987). 
Since $O^{p}(H)$ has no normal subgroup of index $p$, we have $O^{p}(H) \cap P \leq G^{\prime}$. Hence

$$
O^{p}(H) \cap G^{\prime} \cap P=O^{p}(H) \cap P
$$

and $H$ has a normal $p$-complement by Tate's theorem.

Corollary (J. G. Thompson [2]). Suppose that a prime $p$ divides $\chi(1)$ for all nonlinear irreducible characters $\chi$ of $G$. Then $G$ has a normal $p$-complement.

This follows from Theorem, since $B=G$, where $B$ is defined in the theorem.

Remark. We prove that Tate's theorem for $p>2$ is a corollary to the following well-known result of J. G. Thompson [3]:

Let $p>2$, let $P \in \operatorname{Syl}_{p}(G)$, and, for every characteristic subgroup $P_{0}$ of $P, P_{0} \neq 1$, the normalizer $N_{G}\left(P_{0}\right)$ has a normal $p$-complement. Then $G$ has a normal $p$-complement.

Suppose that $H \unlhd G, p>2, P \in \operatorname{Syl}_{p}(G)$, and $P_{1}=H \cap P \leq \Phi(P)$. Suppose that $H$ has no normal $p$-complement. By Thompson's theorem, there exists a characteristic subgroup $P_{0}$ of $P_{1}, P_{0} \neq 1$, such that $N_{H}\left(P_{0}\right)$ has no normal $p$-complement, and let $P_{0}$ have a maximal order among all subgroups with this property. Since $P_{1} \unlhd P$, we have $P_{0} \unlhd P$. So $P<N_{G}\left(P_{0}\right)$. Since $N_{H}\left(P_{0}\right) \leq N_{G}\left(P_{0}\right)$, the subgroup $N_{G}\left(P_{0}\right)$ has no normal $p$-complement. Without loss of generality we may assume that $P H=G$. Then

$$
N_{G}\left(P_{0}\right)=P\left(H \cap N_{G}\left(P_{0}\right)\right)=P N_{H}\left(P_{0}\right)=N_{H}\left(P_{0}\right) P
$$

by modular law. Since $N_{G}\left(P_{0}\right)$ has no normal $p$-complement we may assume without loss that $N_{G}\left(P_{0}\right)=G$. So $P_{0} \unlhd G$. Suppose that $P_{0} \npreceq \Phi(G)$. Then there exists such a maximal subgroup $M$ of $G$ that $P_{0} M=G$. Then $P=$ $P_{0}(P \cap M)$ by modular law. So $P \cap M=P$ (since $\left.P_{0} \leq \Phi(P)\right)$, and $P_{0} \leq M$, $M=P_{0} M=G$, a contradiction. Hence $P_{0} \leq \Phi(G)$. By Thompson's theorem $G / P_{0}$ has a normal $p$-complement $T / P_{0}$ by virtue of a maximal choice of $P_{0}$. If $K$ is a $p^{\prime}$-Hall subgroup of $T$ (Schur-Zassenhaus), then

$$
G=N_{G}(K) T=N_{G}(K) K P_{0}=N_{G}(K) P_{0}
$$

(Schur-Zassenhaus and Frattini). Since $P_{0} \leq \Phi(G)$, we have $N_{G}(K)=G$ and $K \unlhd G$. Obviously, $K$ is a normal $p$-complement of $G$.

Further applications of a generalization of Tate's theorem (Roquette's theorem [4]) can be found in Chapter 6 of the book [5].

\section{ACKNOWLEDGMENT}

The author thanks the referee who suggested a re-statement of the theorem.

\section{REFERENCES}

1. J. Tate, Nilpotent quotient groups, Topology 3 (1964), 109-111.

2. J. G. Thompson, Normal p-complements and irreducible characters, J. Algebra 14 (1970), 129-134. 
3. J. G. Thompson, Normal p-complements for finite groups, Math. Z. 72 (1960), 332-354.

4. P. Roquette, Über die Existenz von Hall-Komplementen in endlichen Gruppen, J. Algebra 1 (1964), 342-346.

5. Ya. G. Berkovich and E. M. Zhmud', Characters of finite groups, Part II (to appear).

Prosp. Engels III, APt. 18, 344006 Rostov-on-Don, USSR 